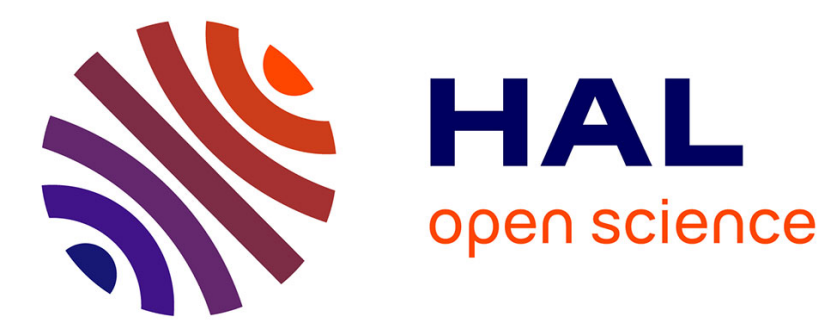

\title{
Towards a biosensor based on Anti Resonant Reflecting Optical Waveguide fabricated from porous silicon.
}

Mohamed Hiraoui, Lazhar Haji, Mohammed Guendouz, Nathalie Lorrain, A. Moadhen, Mehrezi Oueslati

\section{- To cite this version:}

Mohamed Hiraoui, Lazhar Haji, Mohammed Guendouz, Nathalie Lorrain, A. Moadhen, et al.. Towards a biosensor based on Anti Resonant Reflecting Optical Waveguide fabricated from porous silicon.. Biosensors and Bioelectronics, 2012, 36 (1), pp.212-216. 10.1016/j.bios.2012.04.022 . hal-00728030

\section{HAL Id: hal-00728030 https://hal.science/hal-00728030}

Submitted on 4 Sep 2012

HAL is a multi-disciplinary open access archive for the deposit and dissemination of scientific research documents, whether they are published or not. The documents may come from teaching and research institutions in France or abroad, or from public or private research centers.
L'archive ouverte pluridisciplinaire HAL, est destinée au dépôt et à la diffusion de documents scientifiques de niveau recherche, publiés ou non, émanant des établissements d'enseignement et de recherche français ou étrangers, des laboratoires publics ou privés. 


\title{
Towards a biosensor based on Anti Resonant Reflecting Optical Waveguide fabricated from porous silicon.
}

\author{
M. Hiraoui ${ }^{1.2)}$, L. Haji ${ }^{\text {*) }}$, M. Guendouz ${ }^{1)}$, N. Lorrain ${ }^{1)}$, A Moadhen ${ }^{2)}$ and M. Oueslati $^{2)}$ \\ ${ }^{1)}$ Université Européenne de Bretagne, CNRS-UMR FOTON 6082, BP 80518, ENSSAT 6 rue \\ Kerampont, 22305 Lannion Cedex, France. \\ ${ }^{2)}$ Unité de Spectroscopie Raman, Faculté des Sciences de Tunis, 2092 El Manar-Tunisia.
}

\begin{abstract}
Recently, we demonstrated that Anti Resonant Reflecting Optical Waveguide (ARROW) based on porous silicon (PS) material can be used as a transducer for the development of a new optical biosensor. Compared to a conventional biosensor waveguide based on evanescent waves, the ARROW structure is designed to allow a better overlap between the propagated optical field and the molecules infiltrated in the porous core layer and so to provide better molecular interactions sensitivity.

The aim of this work is to investigate the operating mode of an optical biosensor using the ARROW structure. We reported here an extensive study where the antiresonance conditions were adjusted just before the grafting of the studied molecules for a given refractive index range.

The interesting feature of the studied ARROW structure is that it is elaborated from the same material which is the porous silicon obtained via a single electrochemical anodization process. After oxidation and preparation of the inner surface of porous silicon by a chemical functionalization process, bovine serum albumin (BSA) molecules, were attached essentially in the upper layer. Simulation study indicates that the proposed sensor works at the refractive index values ranging from 1.3560 to 1.3655 .

The experimental optical detection of the biomolecules was obtained through the modification of the propagated optical field and losses. The results indicated that the optical attenuation decreases after biomolecules attachment, corresponding to a refractive index change $\Delta \mathrm{n}_{\mathrm{c}}$ of the core. This reduction was of about $2 \mathrm{~dB} / \mathrm{cm}$ and $3 \mathrm{~dB} / \mathrm{cm}$ for Transverse Electric (TE) and
\end{abstract}

\footnotetext{
${ }^{1}$ Electronic mail : mohamed-lazhar.haji@univ-rennes1.fr
} 
Transverse Magnetic (TM) polarizations respectively. Moreover, at the detection step, the optical field was almost located inside the core layer. This result was in good agreement with the simulated near field profiles.

Keywords: Anti Resonant Reflecting Optical Waveguide; Porous silicon; Functionalization; Optical biosensor

\section{Introduction}

Biomolecular detection is of central importance for many potential applications including monitoring of the environment, control of food products and their use in the pharmaceutics industry. Research on biosensors has experienced a noticeable development over the last decades especially in label free optical biosensors which attract a great interest of many scientists (Bier et al., 1996; Brynda et al., 1999; Lin et al., 2002). An interesting review on optical label free biosensors was reported by Xudong Fan et al (Fan et al., 2008) including Surface Plasmon Resonance (SPR) sensor with various configurations. Currently, photonic structures are widely used as transducer for optochemical sensing applications (Jane et al., 2009; Pacholski et al., 2005) based on a change in the effective refractive index due to biomolecules attachment events. Most of them are based on an evanescent wave sensor for optical detection (Parriaux et al., 1995). However, this evanescent wave interacts with biomolecules in remote surface area through exponentially decaying field that only exists within a few hundred nanometers. Then the interaction between biomolecules and the electromagnetic field does not provide optimal result for high sensitivity.

Nowadays, research studies are continuously increasing to find new methods and devices that would provide easy, reproducible and sensitive sensing assays for bio-molecular reactions. PS is an attractive and promising material for biosensing applications because it is cheap, easy to obtain and has a porous structure with a large specific area (up to $800 \mathrm{~m}^{2} / \mathrm{g}$ ) (Herino et al., 1987). Furthermore, this last property makes PS very sensitive to the presence of biochemical elements which penetrate inside the pores.

Many label free optical biosensors based on porous silicon (PS) material have been developed such as waveguides (Rendina et al., 2007; Rong et al., 2008) based on evanescent waves, Bragg mirrors (Snow et al., 1999; Anderson et al., 2003) and microcavities (Chan et al., 2001; De Stefano et al., 2003; Ouyang et al., 2005) based on the shift of the reflectance spectrum peak. In our recent study (Haji et al. 2012), we have demonstrate the feasibility of 
an ARROW structure based on porous silicon for sensing application even that the refractive index contrast is rather low. Thus it can be an interesting candidate for the development of a new optical biosensor in order to increase the available specific surface area and improve the field- biomolecules interaction. The mode of operating of ARROW biosensor is dependent on antiresonance conditions for a determined step of the functionalization process

In this paper, we present an extensive study of a porous silicon based planar ARROW in which the antiresonance conditions are adjusted just before the protein attachment for both Transverse Electric (TE) and Transverse Magnetic (TM) polarizations. The elaboration of the PS material, the functionalization process and the experimental measurements for the optical detection are described. Simulation study was used for the structure design and to predict the ARROW behaviour. Simulated and experimental results are then compared and discussed.

\section{Experimental}

\subsection{Structure of an ARROW}

SEM cross section image of the fabricated porous silica ARROW structure is illustrated in Fig.1a. For this kind of waveguide, the thickness and the refractive index of each porous layer as shown in Fig. 1b must satisfy the antiresonant conditions. The refractive index of the first cladding layer must be higher than those of the core and second cladding layers. Unlike conventional waveguides, in an ARROW structure, the light is confined in the core layer by total internal reflection at the upper air-core layer interface and by anti-resonant reflection due to the presence of the cladding layers underneath the core layer.

For a given thickness of the core layer $d_{c}$, the optimum thicknesses of the two interference cladding layers for the $\mathrm{m}^{\text {th }}$ mode and for the $\mathrm{M}^{\text {th }}$ and $\mathrm{N}^{\text {th }}$ order of the antiresonant conditions satisfy the following relationships (Duguay et al., 1986):

$$
\begin{aligned}
& d_{1} \cong \frac{\lambda}{4 n_{1}}\left(1-\left(\frac{n_{c}}{n_{1}}\right)^{2}+\left(\frac{(m+1) \lambda}{2 n_{1} d_{c}}\right)^{2}\right)^{-1 / 2}(2 M+1) \\
& d_{2} \cong \frac{\lambda}{4 n_{1}}\left(1-\left(\frac{n_{c}}{n_{2}}\right)^{2}+\left(\frac{(m+1) \lambda}{2 n_{2} d_{c}}\right)^{2}\right)^{-1 / 2}(2 N+1)
\end{aligned}
$$

Where $\lambda$ is the light propagation wavelength along the $\mathrm{z}$ direction, $\mathrm{M}$ and $\mathrm{N}=0,1,2 \ldots$ and $\mathrm{m}=0,1,2, \ldots$ In our case, we only have considered the fundamental mode $(\mathrm{m}=0)$. 


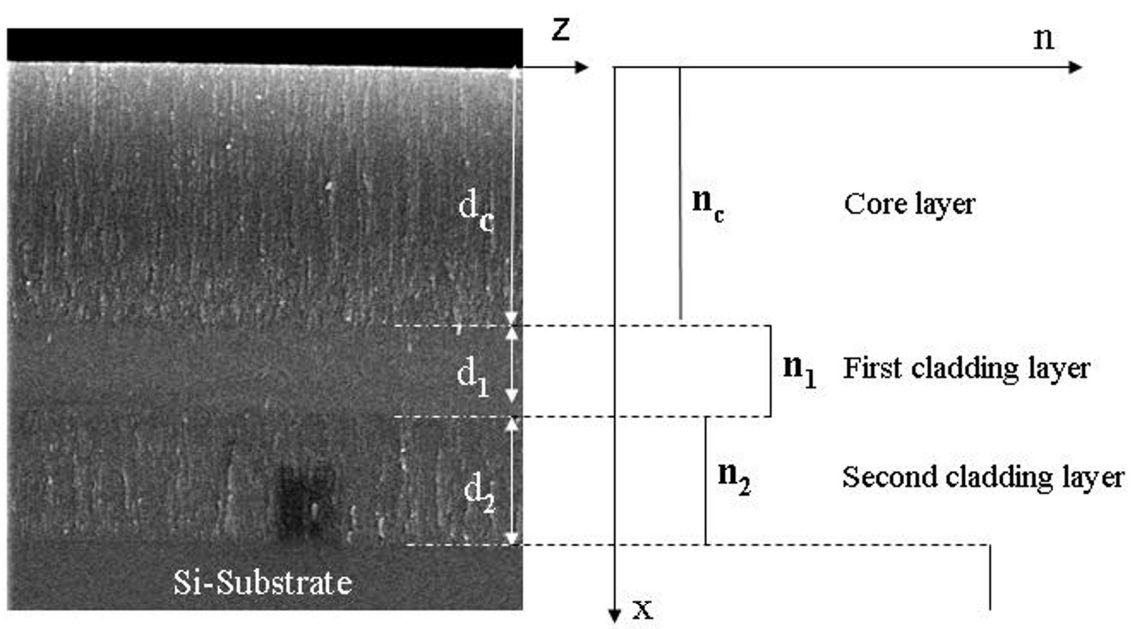

(a)

(b)

Fig. 1 SEM cross section image of the porous silica ARROW structure (a) and refractive index profile (b)

Most of the used methods to elaborate ARROW waveguides are based on technologies using $\mathrm{SiO}_{2} / \mathrm{Si} / \mathrm{SiO}_{2}$ (Shruti et al., 2009), $\mathrm{SiO}_{2} / \mathrm{TiO}_{2} / \mathrm{SiO}_{2}$ (Chen et al., 2008), Polyimide/ $\mathrm{Ta}_{2} \mathrm{O}_{5} /$ Polyimide (Chu and Chuang, 2000) and $\mathrm{SiO}_{2} / \mathrm{Si}_{3} \mathrm{~N}_{4} / \mathrm{SiO}_{2}$ (Prieto et al., 2001; Bernini et al., 2004). In our work, the ARROW structure is elaborated from the same material which is porous silicon obtained by an electrochemical anodization method by varying the current density and the anodizing time.

\subsection{Fabrication}

The guiding structure was elaborated on highly P doped silicon substrate $(7 \mathrm{~m} \Omega . \mathrm{cm})$ by an electrochemical anodization method in an HF solution at room temperature. The electrolyte used was composed of $\mathrm{HF}\left(50 \%\right.$ )-ethanol- $\mathrm{H}_{2} \mathrm{O}$ (deionised water at $18 \mathrm{M} \Omega$,) solution with the ratios of 2-2-1 respectively. By applying different current densities and anodization durations, we can control the porosity and thickness of each PS layer. It is necessary to adjust the electrochemical anodization parameters in order to obtain an upper porous silicon layer (core layer) with pores sufficiently open (mean diameter size of $30 \mathrm{~nm}$ ) which can allow the easy ingress of biomolecules. On the other hand, the first cladding layer (with high refractive index) must have very small pore diameters in order to ensure that this layer is impermeable to molecules. Finally, the second cladding porous layer is made with a refractive index equal 
or greater than the core layer. Moreover, we aimed to obtain roughly the thickness of different layers of the ARROW which satisfy the antiresonant conditions with tolerance on thickness. So a current density of $50 \mathrm{~mA} / \mathrm{cm}^{2}$ was applied for $120 \mathrm{~s}$ to produce the core layer, followed by a current density of $2 \mathrm{~mA} / \mathrm{cm}^{2}$ for $460 \mathrm{~s}$ for the first cladding layer and finally a current density of $19 \mathrm{~mA} / \mathrm{cm}^{2}$ was applied for $110 \mathrm{~s}$ to produce the second cladding layer. After anodization, the structure was then completely oxidized in wet $\mathrm{O}_{2}$ using a two steps thermal treatment $\left(300{ }^{\circ} \mathrm{C}\right.$ for 1 hour and $950{ }^{\circ} \mathrm{C}$ for 1 hour). This oxidation was necessary to obtain transparent porous silica in the visible range for waveguide biosensor applications.

Characteristics of distinct PS layers are reported in table 1, where the thickness, the porosity and the refractive index of each PS layer after the oxidation step are given.

These porosities and refractive indices corresponding to the core layer, the first and second cladding layers were calculated by the Bruggemann model (Aspnes, 1982) at a wavelength of $1.55 \mu \mathrm{m}$.

Table 1

Characteristics of planar ARROW structure based on porous silicon layers after the oxidation.

\begin{tabular}{lccc}
\hline & Thickness $(\mu \mathrm{m})$ & Porosity $(\%)$ & Refractive index \\
\hline Core layer & $3.8 \pm 0.10$ & $32 \pm 6.5$ & $1.298 \pm 0.028$ \\
First cladding layer & $1.4 \pm 0.10$ & $11 \pm 5.0$ & $1.396 \pm 0.023$ \\
Second cladding layer & $2 \pm 0.10$ & $21 \pm 6.5$ & $1.35 \pm 0.028$ \\
\hline
\end{tabular}

\subsection{Functionalization}

The functionalization process was performed using a protocol that we have already developed and tested (Hiraoui et al., 2011) on single layers of porous silicon with thickness and porosity very close to those of the different layers constituting the ARROW structure. The different steps of functionalization were adapted to this type of structure in order to ensure a homogeneous functionalization of the core layer and to prevent any chemical modification of the cladding layers. The in-depth homogeneity was performed by Raman spectroscopy. After the oxidation step, the ARROW structure was immersed in $2 \%(\mathrm{v} / \mathrm{v})$ of 3 Aminopropyltriethoxysilane (APTES) in a hydro-alcoholic solution mixture of Deionised water and methanol (1-1) for 20 minutes at room temperature. Then, the sample was immersed in a $2.5 \%(\mathrm{v} / \mathrm{v})$ solution of Glutaraldehyde (GL) adjusted to $\mathrm{pH} 7$ using sodium hydroxide 
solution for 1 hour at room temperature. Finally, the functionalized ARROW was immersed in a solution of bovine serum albumin with a fixed concentration (BSA, $3 \mathrm{mg} / \mathrm{ml}$ ) at room temperature. The dimensions of BSA molecule are $3 \mathrm{~nm}$ x $8 \mathrm{~nm}$ x $8 \mathrm{~nm}$ (Huang et al., 2004), so the BSA molecules can easily penetrate into the pores (30 $\mathrm{nm}$ mean diameter) after the functionalization process.

\subsection{Optical measurement}

Optical losses are based on a method which consists of measuring the scattered light from the upper surface of the waveguide (Okamura et al., 1983). The probe laser beam (at $1.55 \mu \mathrm{m}$ ) of either TE or TM polarization was coupled into the core layer of the waveguide through a microlensed single-mode optical fiber. Scattered light on the surface was recorded with a digital camera (InGaAs Sensors Unlimited) placed on the top of the surface sample followed by digital image processing. Decreased light intensity is adjusted by an exponential function along the direction of propagation. The slope of this curve corresponds to the attenuation optical coefficient $\alpha\left(\mathrm{cm}^{-1}\right)$ from which we can calculate the optical losses given by the following relation: $\alpha_{d B / c m}=10 \log _{10}(e) \alpha_{c m^{-1}}$

Moreover, the near field profile was recorded through a microscope objective $(40 \mathrm{x})$ placed at the output of the ARROW and the obtained digital image was processed with ImageJ software. It is worth noting that in all the optical characterizations performed here, the ambient conditions (temperature and humidity) were not strictly controlled. However, we do not notice significant changes in the optical characteristics of the ARROW once it was functionalized.

\section{Results and discussion}

\subsection{Sensor mode operation}

The reflectivity coefficient $(\mathrm{R})$ at the interface between the core and the first cladding layers is a key parameter for an ARROW structure. The reflectivity (R) expression for any polarization (TE or TM) is obtained by calculations based on Fresnel coefficients (Brinkmann et al., 2007) and is given by Eq. (4):

$$
R=\left|\frac{r_{c 1}+r_{12} \exp \left(i \phi_{1}\right)}{1+r_{c 1} r_{12} \exp \left(i \phi_{1}\right)}\right|^{2}
$$

With $\phi_{1}=\frac{4 \pi}{\lambda} \cdot n_{1} \cdot d_{1} \cdot \cos \theta_{1}$

And where $r_{i j}$ presents the reflection coefficient at the interface between the layers $i$ and $j(i=$ c or $1 ; \mathrm{j}=1$ or 2 ). 
We present in Fig. 2a the calculated reflectivity for the TE mode as a function of $\mathrm{d}_{1}$ for several core thicknesses. From this figure, we can notice that there are some singular values of $\mathrm{d}_{1}$ where a minimum of reflectivity is obtained, corresponding to resonance values. Whereas, the region of maximum reflectivity corresponds to antiresonance values. It can be also noticed that the reflectivity increases when the core thickness is higher. Therefore, a thicker core layer is preferable to a thinner one in order to obtain a better reflectivity. However, we must always ensure homogeneous infiltration of molecules in the upper active core layer.

Preliminary work was performed on porous silicon single layers to optimize the thickness and refractive index of the different layers of the ARROW structure and to control the variation of the effective refractive index of the active layer after each step of the functionalization process and attachment of protein. Then, we determined the thickness $d_{1}$ for which the antiresonance conditions are satisfied just before the step of protein (BSA) attachment.

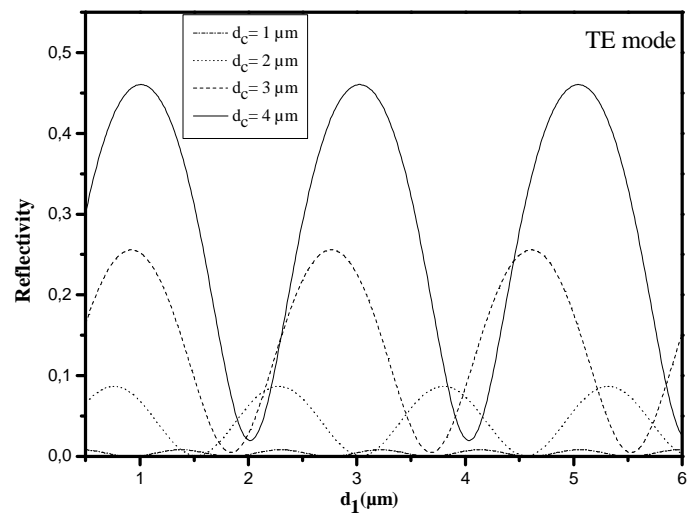

(a)

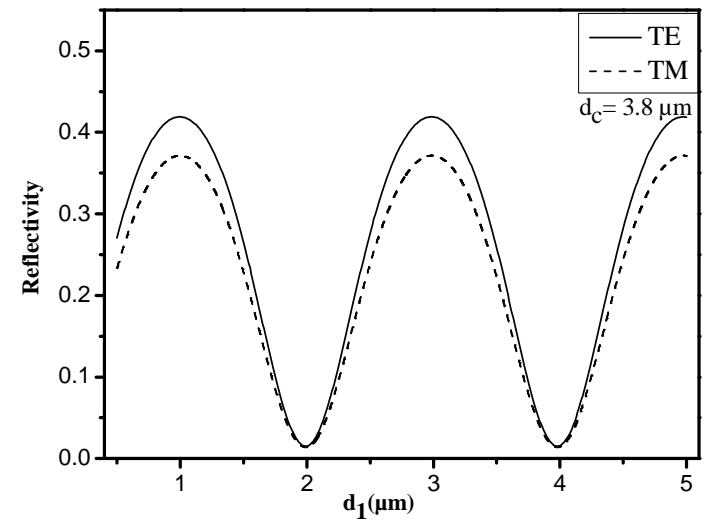

(b)

Fig. 2. Calculated reflectivity as a function of $d_{1}$ (a) for four different thicknesses $d_{c}$ in ARROW structure for the TE mode and (b) for $d_{c}=3.8 \mu \mathrm{m}$ for both TE and TM modes, with refractive index values $\mathrm{n}_{\mathrm{c}}=1.356, \mathrm{n}_{1}=1.396, \mathrm{n}_{2}=1.350$ and working wavelength $\lambda=1.55$ $\mu \mathrm{m}$.

Otherwise in Fig. $2 b$, we can observe that for a thickness $d_{1}$ around $1.2 \mu \mathrm{m}$, the TE mode reflection coefficient is higher than the TM mode reflection coefficient. This antiresonant effect is also obtained for other thicknesses of $d_{1}$ which is identified by a maximum reflectivity with differences between the two polarization modes. On the other hand, when the reflectivity is minimum, for example when the value of $d_{1}$ is around $2 \mu \mathrm{m}$ (or a multiple of 2 $\mu \mathrm{m})$, we do not note any difference in reflectivity between the TE and TM modes. In this case, the resonance conditions are satisfied. The distinction between the TE and TM modes is a feature of an ARROW structure. 
In order to clarify why we have chosen the step just before BSA attachment for which the antiresonance conditions are satisfied, we have plotted (Fig. 3) the calculated reflectivity for the TE and TM modes as a function of the effective refractive index variation of the core layer defined by $\Delta \mathrm{n}_{\mathrm{c}}=\mathrm{n}_{\mathrm{c}}-\mathrm{n}_{\mathrm{c} 0}$. This variation is used as an indication of the presence of the added molecules, where $\mathrm{n}_{\mathrm{c} 0}$ corresponds to the effective refractive index of the core layer just before grafting step. As can be seen in Fig. 3, the presence of two distinct regions with different behaviours can be noticed. For $\Delta \mathrm{n}_{\mathrm{c}} \leq 0$, a weak variation of reflectivity not exceeding 0.4 can be seen and depends on the polarization-mode. Whereas for $\Delta \mathrm{n}_{\mathrm{c}} \geq 0$ and after the coupling step, the reflectivity strongly increases reaching a value of 1 as $\Delta \mathrm{n}_{\mathrm{c}}$ increases and where the ARROW structure acts as a sensor. In addition we notice a slight difference between the two polarization modes TE and TM up to $\Delta \mathrm{n}_{\mathrm{C}}=5.10^{-3}$ and after that the two curves are almost the same. Moreover, this second region is most important for the biosensor sensitivity. Indeed, the sensor's sensitivity can be significantly enhanced while the slope of the curve is high.

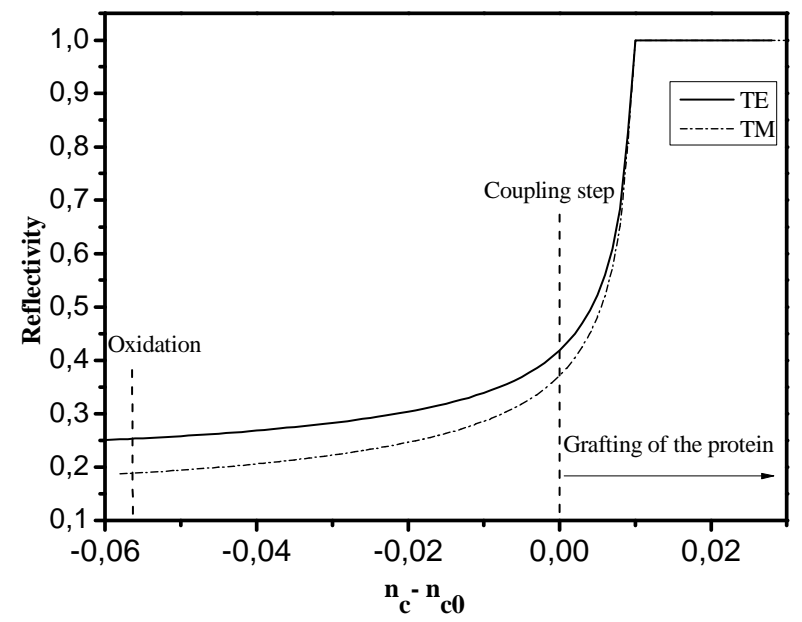

Fig. 3. Calculated reflectivity as a function of the refractive index variation of the core layer after each step (functionalization and BSA grafting), with constant values of $n_{1}$ and $n_{2}$ for TE and TM modes, working wavelength $\lambda=1.55 \mu \mathrm{m}, \mathrm{d}_{\mathrm{c}}=3.8 \mu \mathrm{m}$ and $\mathrm{d}_{1}=1.4 \mu \mathrm{m}$.

Moreover, the attenuation $\alpha$ of the TE and TM fundamental $(m=0)$ ARROW modes, related to the reflectivity (Baba et al., 1988) (defined as the power loss per unit propagation length), is provided by Eq. (6):

$$
\alpha(d B / c m)=2.17(1-R)\left(1 / d_{c}\right)\left[\left(\frac{2 n_{c} d_{c}}{(m+1) \lambda}\right)^{2}-1\right]^{1 / 2}
$$

Thus, according to this relationship, the optical attenuation as a function of the refractive index variation in the core layer will follow the same but opposite behaviour of the one of the 
reflectivity variation which was discussed in the previous section. For $\Delta \mathrm{n}_{\mathrm{c}} \geq 0$, the optical losses decrease rapidly. Such as it has been described in the previous discussion (Fig.3), this decrease is characterized by an insignificant variation between the two polarization modes TE and TM up to $\Delta \mathrm{n}_{\mathrm{C}}=5.10^{-3}$. Similarly for $\Delta \mathrm{n}_{\mathrm{c}} \leq 0$, the optical attenuation weakly decreases and it becomes more dependent on the polarization mode. At the oxidation step, the calculated optical attenuation of TE and TM modes are $2.6 \mathrm{~dB} / \mathrm{cm}$ and $2.9 \mathrm{~dB} / \mathrm{cm}$ respectively. After GL molecules attachment, these calculated attenuation slightly decreases and reaches $2.17 \mathrm{~dB} / \mathrm{cm}$ for TE mode and $2.3 \mathrm{~dB} / \mathrm{cm}$ for TM mode.

\subsection{Protein detection}

We also carried out near-field measurements to study changes in the modal characteristics of the light propagation in the ARROW structure before and after protein binding. In order to verify the feasibility of this oxidized porous silicon based ARROW structure as an optical bio transducer, several simulations were conducted by changing the refractive index of the core layer. The profiles of near field experiments were then compared with those simulated using the Finite Difference-Beam Propagation Method (FD-BPM) and have shown a good agreement. Fig. 4a and Fig. 4c illustrate respectively the simulated and experimental TE field distribution observed at the propagation distance $\mathrm{z}=1 \mathrm{~cm}$. We notice that after oxidation, the light is propagated only in the first cladding layer which has the highest refractive index and this light propagation is similar to the one observed in the case of standard resonant waveguide. We notice that the relatively weak field intensity is due to the fact that light was coupled in that case in the upper sensing layer. We have also attested that if the light is only coupled in the first cladding layer, the maximum field is confined in this layer indicating the resonant behaviour of the waveguide. After GL molecule attachment, we noticed a decrease in the field propagated in the first cladding layer and an appearance of the light guided in the core layer indicating an antiresonant effect. Finally, after BSA attachment, the light is guided almost completely in the core layer. This can be attributed to the increment in the reflection coefficient $\mathrm{R}$ at the interface between the core layer and the first cladding layer. This suggests that BSA molecules were infiltrated into the pores and generated a change in the effective refractive index of the core layer. These results are in good agreement with the simulated reflectivity discussed in the previous section and shown in Fig. 3. As in the case of TEpolarization, it can be noticed that simulated and experimental profiles present an almost similar behaviour for TM-polarization as shown in Fig. 4b,d. However, we observe a 
difference after the GL coupling step which shows the proportionality between the light guided in the core layer and the first cladding layer. This can be explained by the fact that the TM mode has a lower reflection coefficient R compared to the one of the TE mode and then confirms the difference in the propagation light as a function of the mode polarization unlike the case of conventional resonant waveguide.
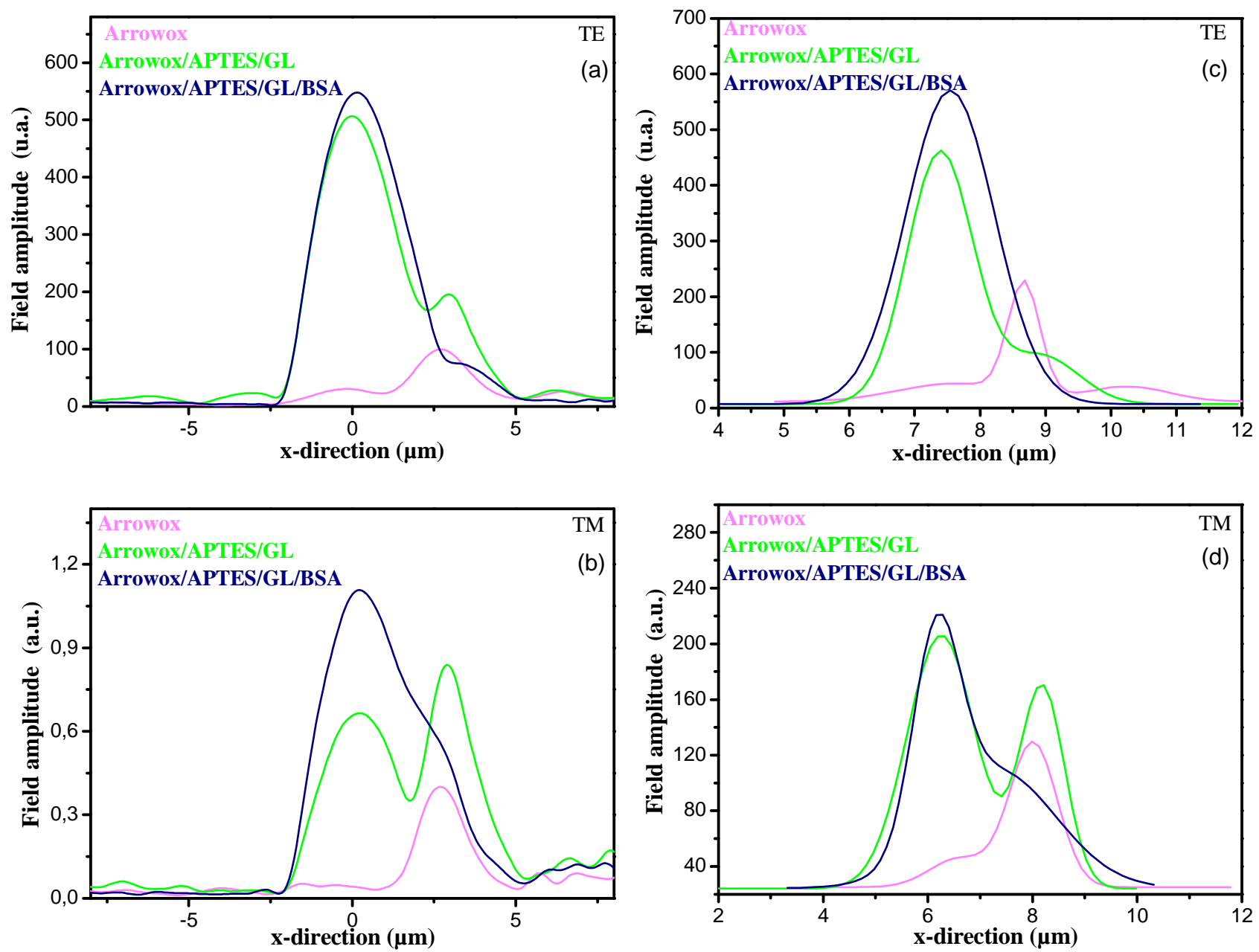

Fig. 4. Near-field profiles: BPM simulated for TE (a) and for TM (b) and experimental for TE (c) and for TM (d).

In addition to near-field measurements, we have also performed optical loss measurements by the method mentioned in section 2.4. In table 2, we have reported the loss values for the ARROW structure before and after protein binding for both TE and TM polarizations.

Table 2

Optical loss measurements of planar ARROW structure after each step: oxidation, functionalization processes (APTES and GL) and BSA attachment for TE and TM polarizations. 


\begin{tabular}{cccc}
\hline & Oxidation (Ox) & Ox/APTES/GL & Ox/APTES/GL/BSA \\
\hline $\boldsymbol{\alpha}(\mathbf{d B} / \mathbf{c m}) \mathbf{T E}$ & $7.5 \pm 0.6$ & $5 \pm 0.4$ & $3 \pm 0.3$ \\
$\boldsymbol{\alpha}(\mathbf{d B} / \mathbf{c m}) \mathbf{T M}$ & $10 \pm 1$ & $7 \pm 0.8$ & $3.7 \pm 0.6$ \\
\hline
\end{tabular}

These results indicate that attenuation decreases when $\Delta \mathrm{n}_{\mathrm{c}}$ increases for the two polarizations. Except for the BSA attachment step, we can also notice clearly that the TM mode attenuation is higher compared to that of the TE mode, which is in concordance with the ARROW features. All these results are in good agreement with the attenuation calculated from simulations but there are differences between the theoretical and experimental values. This discrepancy can be explained by the fact that our calculations do not take into account all the optical losses such as those related to roughness at the interface between the core layer and the first cladding layer.

\section{Conclusion}

In this study, peculiar and interesting properties of a planar ARROW structure based on functionalized porous silica material have been studied. This structure is suitable, in terms of thickness and refractive index of distinct PS layers, to realize label free optical biosensors. The main advantage of using this kind of waveguide based on PS is that it allows a better overlap between the optical field and the molecules attached on the modified porous silica surface since the propagation light is confined inside the upper core layer having the highest porosity therefore the lowest refractive index. Indeed, we have demonstrated the application of the ARROW structure for the detection of biomolecules. In our case, this is obtained through the modification of light propagation modal characteristics and the loss variation in the ARROW structure before and after biomolecules grafting. After proving the feasibility of biosensing using a planar ARROW, the future work consists of integrating this structure in a Mach-Zehnder interferometer for biosensing applications. Furthermore, we will study the analytical performance of the Mach-Zehnder interferometer biosensor by testing different concentration of the analyte. 


\section{References}

Anderson, M.A., Tinsley-Bown, A., Allcock, P., Perkins, E. A., Snow, P., Hollings, M., Smith, R.G., Reeves, C., Squirrell, D.J., Nicklin, S., Cox, T.I., 2003. Phys. Stat. Sol.A. 197, 528-533.

Aspnes, D. E., 1982, Thin Solid Films. 89, 249-262.

Baba, T., Kokubun, Y., Sakaki, T., Iga, K., 1988, Journal of lightwave technology. 6, 14401445 .

Bernini, R., Campopiano, S., Zeni, L., Sarro, P. M., 2004. Sensors and actuators B. 100, 143146.

Bier, F.F., Scheller, F. W., 1996. Biosensors and Bioelectronics. 11, 669-674.

Brinkmann, M., Hayden, J., Letz, M., 2007. Springer handbook of lasers and optics, ed. F. Trager, New York.

Brynda, E., Houska, M., Brandenburg, A., Wikerstal, A., Skvor, J., 1999. Biosensors and Bioelectronics. 14, 363-368.

Chan, S., Li, Yi., Rothberg, L. J., Benjamin Miller, L., Fauchet, F. M., 2001. Materials Science and Engineering C. 15, 277-282.

Chen, C.P., Ma, Z., Anada, T., Hsu, J.P., 2008. Microwave and optical technology letters. 50, 2521-2524.

Chu, A.K., Chuang, M.J., 2000. IEEE Photonics Technology Letters. 12, 1192-1194.

De Stefano, L., Moretti, L., Rendina, I., Rossi, A.M., 2003. Sensors and actuators A. 104, 179-182.

Duguay, M. A., Kokubun, Y., Koch, T.L., 1986, Applied Physics Letters. 49, 13-15.

Fan, X., White, I.M., Shopova, S.I., Zhu, H., Suter, J. D., Sun, Y., 2008. Analytica Chimica. Acta. 620, 8-26.

Haji, L., Hiraoui, M., Lorrain, N., Guendouz, M., 2012, Applied Physics Letters. 100, 111102 .

Herino, R., Bromchil, G., Barla, K., Bertrand, C., 1987, J. Electrochem. Soc. 134, 1994-2000.

Hiraoui, M., Guendouz, M., Lorrain, N., Moadhen, A., Haji, L., Oueslati, M., 2011, Materials Chemistry and Physics. 128, 151-156.

Huang, B. X., Kim, H.Y., Dass, C., 2004. J. Am. Soc. Mass. Spectrum. 15, 1237-1247.

Jane, A., Dronov, R., Hodges, A., Voelcker, N. H., 2009. Trends in Biotechnology. 27, 230239. 
Lin, B., Qiu, J., Gerstenmier, J., Li, P., Pien, H., Pepper, J., Cunningham, B., 2002. Biosensors and Bioelectronics. 17, 827-834.

Okamura, Y., Yoshinaka, S., Yamamoto, S., 1983, Applied Optics. 22, 3892-3894.

Ouyang, H., Christophersen, M., Viard, R., Miller, B. L., Fauchet, P. M., 2005, Advanced Functional Materials. 15, 1851-1859.

Pacholski, C., Sartor, M., Sailor, M. J., Cunin, F., Miskelly, G. M., 2005. J. Am. Chem. Soc. $127,11636-11645$.

Parriaux, O., Sixt, P., 1995. Sensors and Actuators B. 29, 289-292.

Prieto, F., Lechuga, L. M., Calle, A., Llobera, A., Dominguez, C., 2001. Journal of Lightwave Technology, 19, 75-83.

Rong, G., Najmaie, A., Sipe, J. E., Weiss, S. M., 2008. Biosensors and Bioelectronics. 23, 1572-1576.

Shruti, Sinha, R.K., Bhattacharya, R., 2009, Opt Quant Electron. 41, 181-187.

Snow, P.A., Squire, E.K., Russell, P.S.J., Canham, L.T, 1999. J. Appl. Phys. 86, 1781-1784. 